\title{
Variación en el patrón de crecimiento en altura de cuatro especies de Pinus en edades tempranas
}

\author{
Jesús Gustavo Salazar García ${ }^{1}$ \\ J. Jesús Vargas Hernández ${ }^{2}$ \\ Jesús Jasso Mata ${ }^{2}$ \\ José D. Molina Galán ${ }^{3}$ \\ Carlos Ramírez Herrera² \\ Javier López Upton²
}

\begin{abstract}
RESUMEN
Con el propósito de determinar la variación inter e intraespecífica en la tasa de crecimiento en altura y el número de ciclos, se evaluó una plantación experimental de 18 meses de edad, establecida en Patoltecoya, Puebla, durante un periodo de 12 meses. La plantación incluye un total de 30 procedencias de 4 especies de Pinus (10 de $P$. greggii Engelm., 11 de $P$. patula Schl. et Cham., 4 de $P$. tecunumanii (Schw.) Eguiluz et Perry y 5 de $P$. maximinoi $\mathrm{H}$. E. Moore), en un diseño experimental de parcelas divididas en bloques completos al azar, en dos sitios contiguos. No se encontró un efecto significativo del sitio, ni de la interacción genotipo*ambiente en ninguna de las variables del crecimiento evaluadas, pero sí variación significativa ( $p$ $\leq 0.01$ ) entre y dentro de especies en estas características. La variación entre especies fue de 2 a 6 veces mayor que la variación intraespecífica, por lo que existe un mayor potencial de selección a nivel de especies
\end{abstract}

que de procedencias en dichas características. Pinus maximinoi y $P$. tecunumaniipresentaron las mayores tasas de crecimiento en altura ( 2.50 y 2.44 m /año respectivamente), mientras que $P$. patula presentó las menores (1.92 m/año). En $P$. tecunumanii y $P$. patula la tasa de crecimiento se asoció en forma negativa ( $r$ $=-98)$ en la primera y en forma positiva ( $r=$ 0.75 ) en la segunda con el número de ciclos de crecimiento, mientras que en $P$. greggii y $P$. maximinoi no se encontró una correlación significativa entre estas características. Además, se detectaron diferencias importantes entre las especies en el patrón de variación geográfica en las características del crecimiento evaluadas.

\section{PALABRAS CLAVE:}

Crecimiento anual del brote, Pinus greggii, $P$. maximinoi, $P$. patula, $P$. tecunumanii, variación genética.

1 Centro de Investigación Regional del Golfo Centro. INIFAP. Melchor Ocampo No.234 Desp. 313-322, Zona Centro. 91700, Veracruz, Veracruz.

2 Especialidad de Postgrado Forestal. IRENAT. Colegio de Postgraduados. 56230, Montecillo, Edo. de México. Correo electrónico: vargashj@colpos.colpos.mx.

3 Especialidad de Postgrado Genética. IRGP. Colegio de Postgraduados. 56230, Montecillo, Edo. de México. Manuscrito recibido para su publicación el 16 de febrero de 1999 


\section{ABSTRACT}

In order to determine the level of inter and intraspecific variation in height growth rate and number of growth cycles, an 18months old experimental plantation established at Patoltecoya, Puebla was evaluated during a 12-month period. The plantation includes a total of 30 provenances of four Pinus species (10 of $P$. greggii Engelm., 11 of $P$. patula Schl. et Cham., 4 of $P$. tecunumanii (Schw.) Eguiluz et Perry and 5 of $P$. maximinoi $\mathrm{H}$. E. Moore), in a split-plot random block experimental design on two adjacent sites. Both site and site* genotype interaction had no significant effect on growth variables, but species and provenances within species showed a significant variation $(p \leq 0.01)$ in all variables. Variation among species was 2 to 6 times higher than within species, so there is a greater selection potential at the species level for all variables measured. Pinus maximinoi and $P$. tecunumanii showed the highest growth rate (2.50 and $2.44 \mathrm{~m}$ year $^{-1}$ respectively) while $P$. patula presented the lowest growth rate $\left(1.92 \mathrm{~m}_{\text {year }}{ }^{-1}\right)$. Height growth was negatively correlated $(r=-0.98)$ with the number of growth cycles in $P$. tecunumanii and positively correlated $(r=$ 0.75 ) in $P$. patula, but no significant correlation was found between these traits in $P$. greggii and $P$. maximinoi. In addition, each species showed a particular geographic variation pattern in these growth traits.

KEY WORDS:

Shoot growth, Pinus greggii, P. maximinoi, $P$. patula, $P$. tecunumanii, genetic variation.

\section{INTRODUCCION}

El crecimiento en altura de las especies de pino se caracteriza por presentar varios elementos que nos ayudan a identificar y separar el crecimiento del brote líder en componentes. Los componentes más evidentes del brote son el número y longitud de los ciclos de crecimiento que se forman durante las condiciones ambientales favorables para su desarrollo en el año (Cannell et al., 1976).

El número y longitud de los ciclos de crecimiento que se forman en un año dependen del patrón de crecimiento de la especie que encuentra genéticamente controlado (Bridgewater et al., 1985), y estos componentes parecen heredarse de manera independiente (Cannell et al., 1976). Los componentes del crecimiento en altura influyen sobre la productividad al afectar la tasa de crecimiento en altura y la calidad de la madera al afectar la cantidad o proporción de ramas y nudos en el tronco de árboles jóvenes (Daniel et al., 1982; Zobel y Buijtenen, 1989); esta característica es especialmente importante en $P$. patula debido a que tiende a conservar sus ramas por mucho tiempo (Loock, 1950), aún en densidades de plantación elevadas (Wormald, 1975). Los componentes del crecimiento en altura también son un resultado de la capacidad de adaptación de los individuos a condiciones ambientales específicas del sitio de plantación. La capacidad de adaptación es importante porque al establecer plantaciones con especies ó poblaciones fuera de su ambiente natural, como es el caso de la mayoría de las procedencias del presente estudio, los árboles responden a las condiciones del nuevo ambiente, ya sea adaptándose con altos ó bajos rendimientos de madera ó no adaptándose y muriendo (Zobel y Talbert, 1988). Debido a lo anterior, es necesario realizar estudios del nivel de variación genética existente en los componentes del crecimiento y su relación con la tasa de crecimiento anual en altura en especies de Pinus de importancia en plantaciones comerciales en México. Dichos estudios proporcionan información tanto del potencial productivo de las especies como de las posibilidades de adaptación a condiciones ambientales específicas en el sitio de plantación. 
En México existe un número elevado de especies de Pinus que tienen potencial para ser utilizadas en plantaciones forestales comerciales en ambientes diversos. Dentro del grupo de especies subtropicales de Pinus destacan $P$. maximinoi H.E. Moore, $P$. tecunumanii (Schw.) Eguiluz et Perry, P. patula Schl. et Cham. y $P$. greggii Engelm., que han presentado tasas elevadas de crecimiento en altura en edades jovenes al ser evaluadas fuera de su ambiente natural (Dvorak, et al., 1995; Dvorak y Donahue, 1988; Dvorak y Donahue, 1992). Sin embargo, no se tiene información suficiente del patrón de crecimiento anual en estas especies ni de sus implicaciones sobre la tasa de crecimiento en altura en edades juveniles.

En un estudio aislado, López (1998) encontró una amplia variación genética entre y dentro de poblaciones de $P$. greggii Engelm. en la tasa de crecimiento en altura, asociada fundamentalmente a diferencias en el número y longitud promedio de los ciclos de crecimiento. En otros estudios realizados con especies subtropicales de Pinus, como $P$. taeda L. y $P$. patula Schl. et Cham., también se han encontrado resultados similares (Allen y Wentworth, 1993; Gómez et al., 1998), aunque en algunos casos se ha encontrado que no existe una relación significativa entre el crecimiento anual en altura y alguno de sus componentes (Vargas y Jacob, 1993). Lo anterior implica que la importancia relativa de los componentes del crecimiento en altura puede variar de una especie a otra 0 incluso entre poblaciones dentro de una misma especie.

\section{OBJETIVOS}

El presente trabajo tiene como objetivo determinar el nivel de variación genética inter e intraespecífica en la tasa de crecimiento en altura de cuatro especies subtropicales del género Pinus nativas de México y Centroamérica (Pinus greggii
Engelm., Pinus patula Schl. et Cham., Pinus maximinoiH. E. Moore y Pinus tecunumanii (Schw.) Eguiluz et Perry), y su relación con el número de ciclos de crecimiento, así como el posible patrón geográfico de variación de las variables mencionadas dentro de cada especie. Estas especies son comúnmente empleadas en el establecimiento de plantaciones forestales comerciales para la producción de material celulósico y madera aserrada.

\section{METODOLOGÍA}

La plantación experimental se ubica en la finca "Los Ayacahuites" (20/13' latitud N y 98/ 03' longitud W), en el Km 102.6 de la carretera federal Pachuca-Tuxpan, en el poblado de Patoltecoya, Huauchinango, Puebla. La plantación se sitúa en un terreno forestal, con una pendiente de $60 \%$ y con exposición Sur. La altitud aproximada es de 1440 metros sobre el nivel del mar. Se tiene un clima templado lluvioso (García, 1981), con una temperatura media anual de 15.9/ C y una precipitación anual promedio de $2534 \mathrm{~mm}$. El ensayo experimental incluye un total de 30 procedencias de cuatro especies de pinos subtropicales: 10 de Pinus greggii, 5 de $P$. maximinoi, 11 de $P$. patula y 4 de $P$. tecunumanii. Las procedencias representan una proporción diferente de la distribución natural de cada especie (Tabla 1).

La plantación se estableció en el mes de julio de 1995 en cepa común a un espaciamiento de $3 \times 3 \mathrm{~m}$. El terreno se dividió en dos zonas a lo largo de la pendiente; la parte alta del terreno se consideró de menor fertilidad que la parte baja del mismo. Cada zona se consideró como un sitio diferente. El diseño experimental fue parcelas divididas en bloques completos al azar con cuatro repeticiones. Cada repetición se acomodó en forma transversal a la pendiente. En las parcelas grandes se asignaron las especies en forma aleatoria y en las parcelas chicas se ubicaron las procedencias de cada 
especie en forma sistemática. Las procedencias estuvieron representadas por cinco árboles plantados en línea en cada parcela.

Las variables analizadas fueron el crecimiento anual en altura medido en centímetros y el número de ciclos de crecimiento formados durante el periodo de enero de 1997 a enero de 1998. La edad de la plantación al final del periodo de evaluación fue de 2.5 años (julio de 1995 a enero de 1998). No se evaluaron 110 árboles que presentaron el ápice cortado o bifurcado.

El inicio del crecimiento anual en altura se señaló con una cinta colocada en el tronco, para medir a partir de ésta hasta el ápice del árbol. En el caso del número de ciclos de crecimiento se contaron todos los verticilos formados en el eje central desde el punto donde se colocó la cinta hasta el brote terminal. Los datos se analizaron estadísticamente con el siguiente modelo:

$$
\begin{aligned}
\mathrm{Y}_{\mathrm{ijklm}}= & \mu+\mathrm{S}_{\mathrm{i}} * \mathrm{~B}_{\mathrm{j}}\left(\mathrm{S}_{\mathrm{i}}\right)+\mathrm{E}_{\mathrm{k}}+\mathrm{S}_{\mathrm{i}} * \mathrm{E}_{\mathrm{k}}+ \\
& \mathrm{B}_{\mathrm{j}}\left(\mathrm{S}_{\mathrm{i}}\right) * \mathrm{E}_{\mathrm{k}}+\mathrm{P}_{1}\left(\mathrm{E}_{\mathrm{k}}\right)+\mathrm{S}_{\mathrm{i}} * \mathrm{P}_{1}\left(\mathrm{E}_{\mathrm{k}}\right)+ \\
& \mathrm{B}_{\mathrm{j}}\left(\mathrm{S}_{\mathrm{i}}\right) * \mathrm{P}_{1}\left(\mathrm{E}_{\mathrm{k}}\right)+\mathrm{W}_{\mathrm{ijklm}}
\end{aligned}
$$

Donde:

$S=$ Sitio

$\mathrm{B}=$ Bloque

$E$ = Especie

$\mathrm{P}=$ Procedencia

$\mathrm{W}=$ Error aleatorio.

Los efectos de las especies y las procedencias se consideraron como efectos aleatorios con el propósito de evaluar el nivel de variación inter e intraespecífica, mientras que los efectos de sitio y de bloque se consideraron fijos. De acuerdo al diseño experimental, esto generó un modelo mixto, que incluye factores cruzados y anidados, con efectos fijos y aleatorios. Los efectos de especies y procedencias así como los efectos de sitios y la interacción sitio por especies y procedencias se determinó de acuerdo a la esperanzas de los cuadrados medios respectivos que se muestran en la Tabla 2.

Debido al desbalance en el diseño experimental ocasionado por las diferencias en el número de procedencias por especie y número de árboles vivos por parcela, los componentes de varianza se estimaron en forma aproximada con base en los coeficientes $\left(\mathrm{k}_{\mathrm{i}}\right)$ asociados a cada componente en la estructura de cuadrados medios.

Además, con los datos promedio por procedencia se realizó un análisis de correlación en cada especie con el propósito de establecer las posibles correlaciones entre las variables estudiadas a nivel de procedencias, así como para establecer la presencia de posibles patrones geográficos de variación dentro de cada especie.

\section{RESULTADOS Y DISCUSION}

\section{Componentes de varianza.}

El análisis de varianza demostró que no existen diferencias significativas ( $\mathrm{p} \leq$ 0.05 ) entre los sitios en ninguna de las variables del crecimiento incluidas en el estudio (Tabla 3). De la misma manera, tampoco se encontró un efecto significativo de la interacción sitio*especie y sitio*procedencia dentro de especies. Esto implica que no existe una interacción significativa genotipo*ambiente en estas características, por lo que el comportamiento de las especies y procedencias se mantuvo estable de un sitio a otro. Lo anterior se explica debido a la cercanía de los sitios.

A nivel de especies y de procedencias dentro de ellas se encontró una variación altamente significativa $(p \leq 0.01)$ en el crecimiento anual en altura y en el número de ciclos de crecimiento (Tabla 3 ). 
Tabla 1. Coordenadas geográficas (latitud, longitud y altitud) de las especies y de las procedencias utilizadas en el estudio

\begin{tabular}{|c|c|c|c|c|}
\hline Especie & Procedencia & Latitud $(\mathrm{N})$ & Longitud (W) & Altitud (msnm) \\
\hline \multirow[t]{11}{*}{ Pinus greggii } & & $20 /$ a $26 /$ & 97/ a 101/ & 1300 a 3000 \\
\hline & El Madroño, Qro. & $21 / 17$ & 99/ 10' & 1740 \\
\hline & Valle Verde, Qro. & $21 / 16^{\prime}$ & $99 / 12$ & 1600 \\
\hline & Laguna Seca, Hgo. & $21 / 01$ & 99/ 06' & 1720 \\
\hline & San Joaquín, Qro. & $20 / 56^{\prime}$ & $99 / 34$ & 2250 \\
\hline & El Piñón, Hgo. & $20 / 56^{\prime}$ & $99 / 12$ & 1850 \\
\hline & Molango, Hgo. & $20 / 50^{\prime}$ & $98 / 44^{\prime}$ & 1300 \\
\hline & Xochicoatlán, Hgo. & $20 / 50$ & $98 / 43$ & 1920 \\
\hline & Cieneguilla, Hgo. & $20 / 44^{\prime}$ & $99 / 02$ & 2080 \\
\hline & Zimapán, Hgo. & $20 / 43^{\prime}$ & $99 / 23$ & 1850 \\
\hline & Patoltecoya, Pue. & $20 / 13^{\prime}$ & 98/ 03' & 1440 \\
\hline \multirow[t]{5}{*}{ Pinus maximinoi } & \multirow{5}{*}{$\begin{array}{l}\text { Altamirano, Chis., Méx. } \\
\text { San Jerónimo, Oax., Méx. } \\
\text { San Juan Sacatepequez, } \\
\text { Guatemala } \\
\text { Tatumbla, Fco. Morazán, } \\
\text { Honduras } \\
\text { San José Bayancuch, } \\
\text { Nicaragua }{ }^{\dagger}\end{array}$} & $13 /$ a $24 /$ & 97/ a 107/ & 600 a 2400 \\
\hline & & $\begin{array}{l}16 / 25 \\
16 / 06\end{array}$ & $\begin{array}{l}\text { 92/ 01' } \\
\text { 97/ 00' }\end{array}$ & $\begin{array}{l}1350 \\
1480\end{array}$ \\
\hline & & $14 / 24$ & $90 / 22$ & 2000 \\
\hline & & $14 / 12$ & $87 / 04$ & 1600 \\
\hline & & $13 / 23^{\prime}$ & $86 / 17$ & 1500 \\
\hline \multirow[t]{12}{*}{ Pinus patula } & & 17/ a 24/ & $96 /$ a $100 /$ & 1500 a 3100 \\
\hline & Pinal de Amoles, Qro. & 21/ 01' & $99 / 10$ & 2400 \\
\hline & Encarnación, Hgo. & $20 / 31$ & 99/ 07' & 2400 \\
\hline & Zacualtipan, Hgo. & 20/ 23' & $98 / 24$ & 2220 \\
\hline & Tlahuelompan, Hgo. & 20/ 23' & $98 / 21$ & 2020 \\
\hline & Huayacocotla, Ver. & $20 / 19^{\prime}$ & $98 / 17$ & 2030 \\
\hline & Estación Apulco, Hgo. & $20 / 13$ & $98 / 13$ & 2190 \\
\hline & Acaxochitlán, Hgo. & 20/ 06' & $98 / 07$ & 2290 \\
\hline & Ahuazotepec, Pue. & 20/ 00' & $98 / 07$ & 2460 \\
\hline & Zacatlán, Pue. (Nte.) & 19/34' & $97 / 35$ & 2000 \\
\hline & Zacatlán, Pue. & 19/ 33' & $97 / 34$ & 2360 \\
\hline & Tlaxco, Tlax. & $19 / 22$ & 98/ 04' & 2800 \\
\hline \multirow[t]{4}{*}{ Pinus tecunumanii } & & $13 /$ a 17/ & $87 /$ a 92/ & 1500 a 2600 \\
\hline & Chanal, Chis., Méx. & $16 / 25$ & 92/ 13' & 2350 \\
\hline & \multirow{2}{*}{$\begin{array}{l}\text { Rancho Nuevo, Chis., Méx. } \\
\text { San Esteban, Honduras } \\
\text { Yucul, Matagalpa, } \\
\text { Nicaragua }\end{array}$} & $\begin{array}{l}16 / 24 \\
15 / 09\end{array}$ & $\begin{array}{l}92 / 21 \\
85 / 22\end{array}$ & $\begin{array}{l}2340 \\
1200\end{array}$ \\
\hline & & 12/ 33' & $85 / 38^{\prime}$ & 1170 \\
\hline
\end{tabular}

${ }^{\dagger}$ Datos estimados de una carta topográfica. Datos de distribución de las especies tomados de Perry (1991). 
Tabla 2. Esperanza de cuadrados medios para el modelo estadístico de las variables de altura y número de ciclos

\begin{tabular}{|c|c|c|c|}
\hline $\begin{array}{l}\text { FUENTE DE } \\
\text { VARIACION }\end{array}$ & G.L. & & ESPERANZA DE CUADRADOS MEDIOS \\
\hline Sitio & $s-1$ & $\mathrm{CM}_{\mathrm{S}}$ & $\begin{aligned}= & \sigma^{2}{ }_{w}+k_{16} \sigma_{B(S) *(E)}^{2}+k_{17} \sigma_{S^{*} P(E)}^{2}+k_{18} \sigma_{B(S)^{*} E}^{2}+k_{19} \sigma_{S^{*} E}^{2}+ \\
& k_{20} \Sigma[B(S)]^{2} / s(b-1)+k_{21} \Sigma S^{2} / s-1\end{aligned}$ \\
\hline Bloque(Sitio) & $s(b-1)$ & $\mathrm{CM}_{\mathrm{B}(\mathrm{S})}$ & $=\sigma^{2}{ }_{\mathrm{w}}+\mathrm{k}_{13} \sigma_{\mathrm{B}(\mathrm{S})^{*} \mathrm{P}(\mathrm{E})}^{2}+\mathrm{k}_{14} \sigma_{\mathrm{B}(\mathrm{S})^{*} \mathrm{E}}^{2}+\mathrm{k}_{15} \Sigma[\mathrm{B}(\mathrm{S})]^{2} / \mathrm{s}(\mathrm{b}-1)$ \\
\hline Especie & $e-1$ & $\mathrm{CM}_{\mathrm{E}}$ & $\begin{aligned}= & \sigma^{2}{ }_{w}+k_{7} \sigma_{B(S)^{*}(E)}^{2}+k_{8} \sigma_{S^{*} P(E)}^{2}+k_{11} \sigma_{P(E)}^{2}+k_{9} \sigma_{B(S)^{*} E}^{2}+ \\
& k_{10} \sigma_{S^{*} E}^{2}+k_{12} \sigma_{E}^{2}\end{aligned}$ \\
\hline$S^{*} E$ & $(\mathrm{~s}-1)(\mathrm{e}-1)$ & $\mathrm{CM}_{\mathrm{S}^{*} \mathrm{E}}$ & $=\sigma^{2}{ }_{w}+k_{7} \sigma_{B(S)^{*} P(E)}^{2}+k_{8} \sigma_{S^{*} P(E)}^{2}+k_{9} \sigma_{B(S)^{*} E}^{2}+k_{10} \sigma_{S^{*} E}^{2}$ \\
\hline$B(S)^{\star} E$ & $s(b-1)^{\star}(e-1)$ & $\mathrm{CM}_{\mathrm{B}(\mathrm{S})^{*} \mathrm{E}}$ & $=\sigma^{2}{ }_{w}+k_{5} \sigma_{B(S)^{*} P(E)}^{2}+k_{6} \sigma_{B(S)^{*} E}^{2}$ \\
\hline Proc(E) & $\Sigma\left(\mathrm{p}_{\mathrm{i}}-1\right)$ & $\mathrm{CM}_{\mathrm{P}(\mathrm{E})}$ & $=\sigma^{2}{ }_{W}+k_{2} \sigma_{B(S)^{*}(E)}^{2}+k_{3} \sigma_{S^{*} P(E)}^{2}+k_{4} \sigma_{P(E)}^{2}$ \\
\hline$S^{*} P(E)$ & $(\mathrm{s}-1)^{\star} \Sigma\left(\mathrm{p}_{\mathrm{i}}-1\right)$ & $\mathrm{CM}_{\mathrm{S}^{*} \mathrm{P}(\mathrm{E})}$ & $=\sigma^{2}{ }_{w}+k_{2} \sigma_{B(S)^{*} P(E)}^{2}+k_{3} \sigma_{S^{*} P(E)}$ \\
\hline$B(S){ }^{\star} P(E)$ & $\mathrm{s}(\mathrm{b}-1)^{\star} \Sigma\left(\mathrm{p}_{\mathrm{i}}-1\right)$ & $\mathrm{CM}_{\mathrm{B}(\mathrm{S})^{*} \mathrm{P}(\mathrm{E})}$ & $=\sigma^{2}{ }_{w}+k_{1} \sigma_{B(S)^{*}(E)}^{2}$ \\
\hline $\begin{array}{l}\text { Error de } \\
\text { Muestreo }\end{array}$ & por diferencia & $\mathrm{CM}_{\mathrm{w}}$ & $=\sigma_{w}^{2}$ \\
\hline
\end{tabular}

donde: $\mathrm{E}=$ Especie, $\mathrm{S}=$ Sitio, $\mathrm{B}=$ Bloque, $\mathrm{P}=$ Procedencia, $\mathrm{S}=$ No. de sitios, $\mathrm{b}=$ No. de bloques, $\mathrm{e}=\mathrm{No}$. de especies, $\mathrm{p}_{\mathrm{i}}=$ No. de procedencias en la especie $\mathrm{i}, \mathrm{k}_{\mathrm{i}}=$ Coeficiente de cada componente de varianza.

Tabla 3. Cuadrados medios del análisis de varianza de las variables de altura y número de ciclos de crecimiento

\begin{tabular}{|c|c|c|c|}
\hline \multirow[b]{2}{*}{ FUENTE DE VARIACION } & \multirow[b]{2}{*}{ G. L. } & \multicolumn{2}{|c|}{ "CUADRADOS MEDIOS } \\
\hline & & Crecimiento en altura & $\begin{array}{c}\text { Número de ciclos de } \\
\text { crecimiento }\end{array}$ \\
\hline Sitios & 1 & 236212.20 n.s. & 7.55 n.s. \\
\hline Bloques (Sitios) & 6 & 12341.03 & 4.81 \\
\hline Especies & 3 & $180323.5^{* *}$ & $218.25^{* \star}$ \\
\hline Sitios*Especies & 3 & 3337.95 n.s. & 3.90 n.s. \\
\hline Bloques $(S)^{\star}$ Especies & 18 & 14592.6 & 2.54 \\
\hline Proc. (Especies) & 26 & $10333.42^{* *}$ & $8.83^{* *}$ \\
\hline Sitios*Proc.(E) & 26 & 1778.12 n.s. & 2.23 n.s. \\
\hline Bloques(S)*Proc.(E) & 156 & 2910.43 & 1.92 \\
\hline Error & 850 & 1885.91 & 1.99 \\
\hline
\end{tabular}


Esto deja en claro que existe una variación genética inter e intraespecífica importante en las cuatro especies de Pinus con relación a las variables mencionadas.

Aunque no existen referencias de estudios en donde se han evaluado estas cuatro especies en un mismo sitio, sí se ha detectado anteriormente una variación intraespecífica significativa en el crecimiento en altura en edades tempranas de poblaciones de $P$. maximinoi, y $P$. tecunumanii (Dvorak y Donahue, 1992; Wright y Osorio, 1992), $P$. patula (Dvorak et al., 1995) y $P$. greggii (Dvorak et al., 1996; López, 1998) plantadas en diferentes regiones de México, Colombia, Brasil y Sudáfrica.

Las especies aportaron una mayor proporción de la variación fenotípica total encontrada en el ensayo que las procedencias. En el caso del crecimiento en altura las especies contribuyeron con un poco más de dos veces de variación que las procedencias dentro de cada especie (18.8 vs $8.41 \%$ ) a la variación total observada; de la misma manera, en el número de ciclos de crecimiento la variación interespecífica fue casi 6 veces mayor ( 30.23 vs $5.2 \%$ ) que la variación intraespecífica (Fig. 1). Lo anterior muestra que existe una clara diferenciación entre las especies en la tasa y el patrón estructural de crecimiento del brote terminal y que a pesar de la variación intraespecífica existente en cada una de ellas es posible identificar en este grupo de especies de Pinus subtropicales algunas con mayor potencial productivo y con características distintivas en la estructura del crecimiento en altura del tallo.

Lo anterior implica que dentro de este grupo de especies existe un mayor potencial de selección a nivel de especies que a nivel de procedencias en cuanto a la tasa de crecimiento en altura en edades tempranas. Las diferencias en el nivel de variación entre especies y entre procedencias es más notable para el número de ciclos de crecimiento, que para el crecimiento en altura. Por lo tanto, se pueden tener resultados favorables en relación a la calidad de la madera al seleccionar especies que desarrollen un menor número de ciclos de crecimiento y que mantengan tasas elevadas de crecimiento en altura.

\section{Variación interespecífica.}

La tasa de crecimiento promedio anual en altura varió desde $1.92 \mathrm{~m}$ en $P$. patula hasta $2.50 \mathrm{~m}$ en $P$. maximinoi; $P$. greggii y $P$. tecunumanii presentaron valores promedio de 2.19 y 2.44 m, respectivamente (Fig. 2). Considerando estos valores, el crecimiento en altura de $P$. maximinoi es $30 \%$ mayor que el de $P$. patula. A pesar de que Pinus greggii generalmente tiene crecimientos menores que $P$. patula, ya que la primera procede de ambientes más secos y de mayor elevación (Perry, 1991), en este caso tuvo una tasa de crecimiento anual promedio $14 \%$ mayor que la de $P$. patula. Es importante señalar que aunque $P$. patula tuvo la menor tasa de crecimiento promedio anual de las cuatro especies evaluadas, el crecimiento en altura en el año fue de casi dos metros (1.92 m), lo cual no es nada despreciable, comparado con otras especies de pino que tienen tasas de crecimiento mucho menores. Además, es posible que el menor crecimiento de $P$. patula esté relacionado con las desventajas iniciales de la especie ya que la planta tenía una menor edad (3 meses menor) al momento de establecer la plantación. Por otro lado, es posible que las diferencias en las tasas de crecimiento en altura observadas entre las especies no se mantengan al pasar el tiempo, debido a que las especies pueden modificar su tasa y su curva de crecimiento en altura con la edad (Zobel y Talbert, 1988). 


\section{a) Crecimiento en altura}

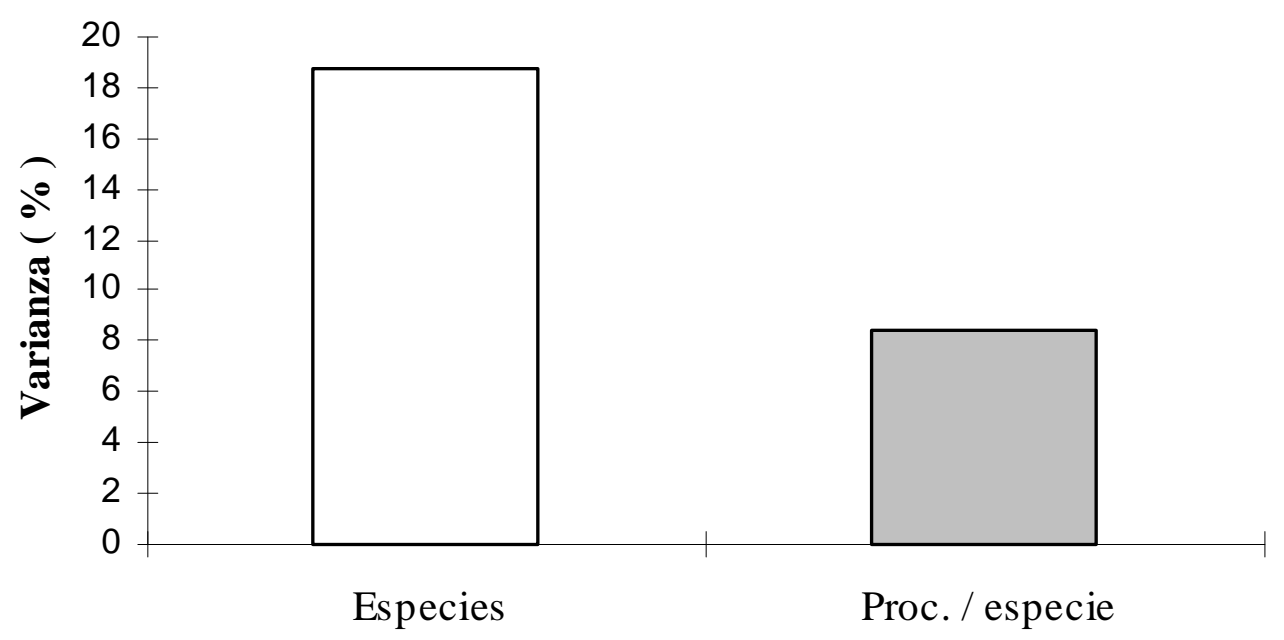

b) Número de ciclos de crecimiento

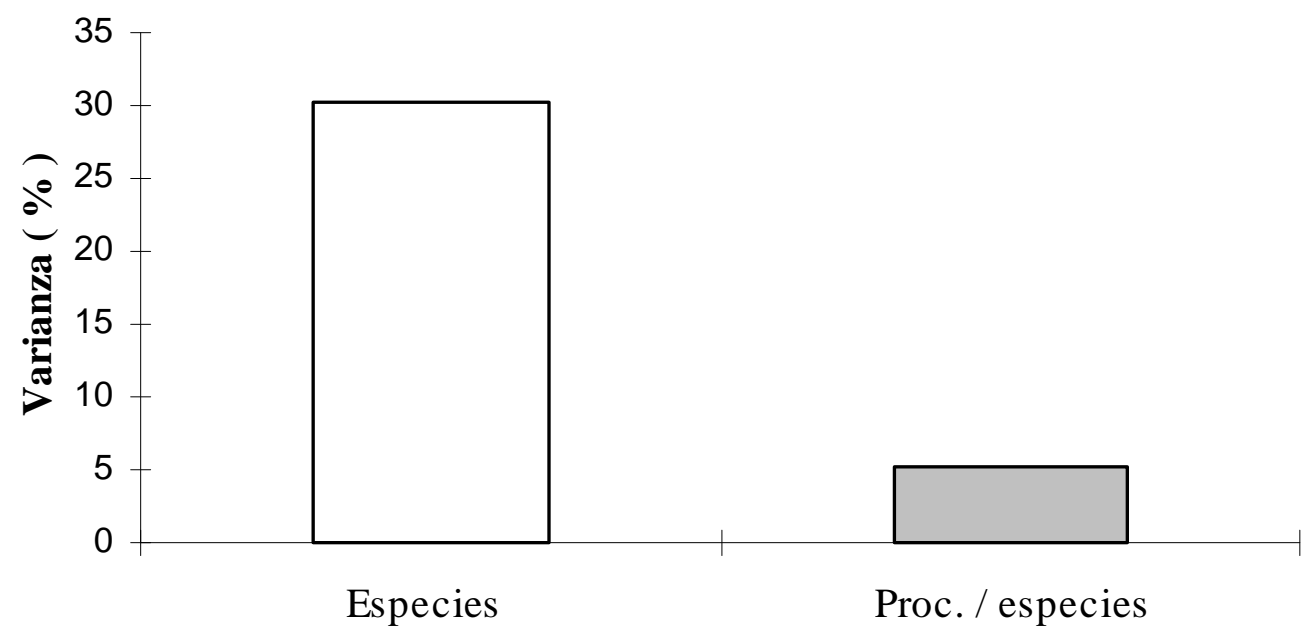

Figura 1. Componentes de varianza (\%) entre especies y entre procedencias dentro de especies en el crecimiento en altura (a) y número de ciclos de crecimiento (b). 
a) Crecimiento en altura

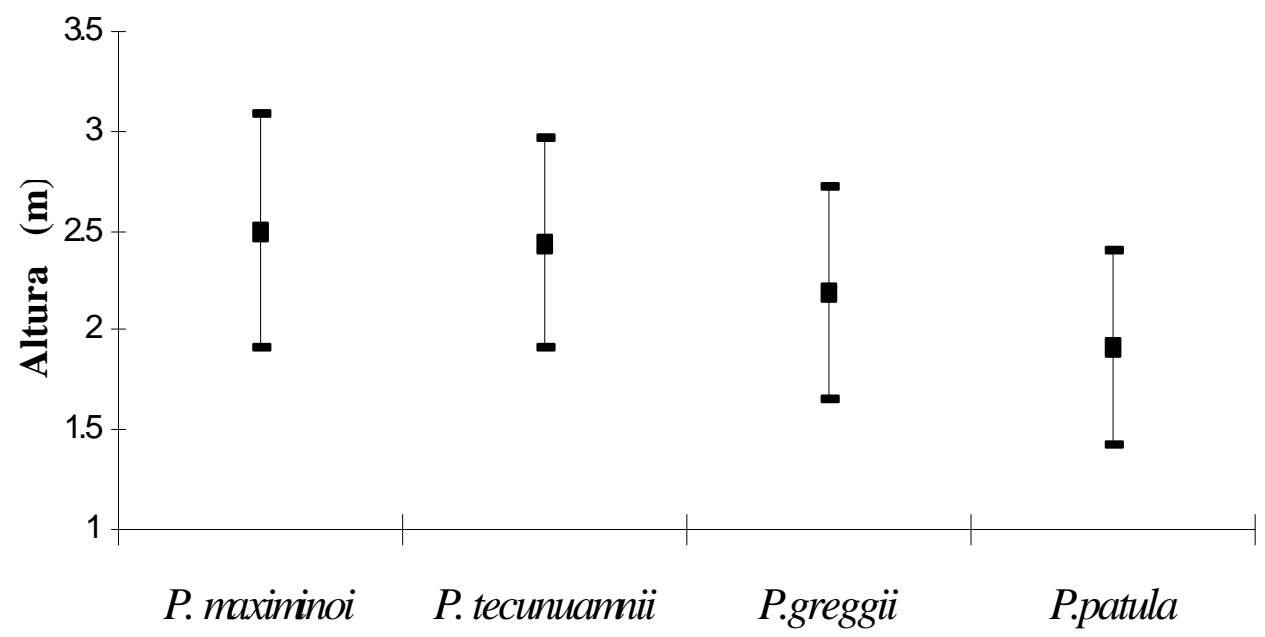

b) Número de ciclos de crecimiento

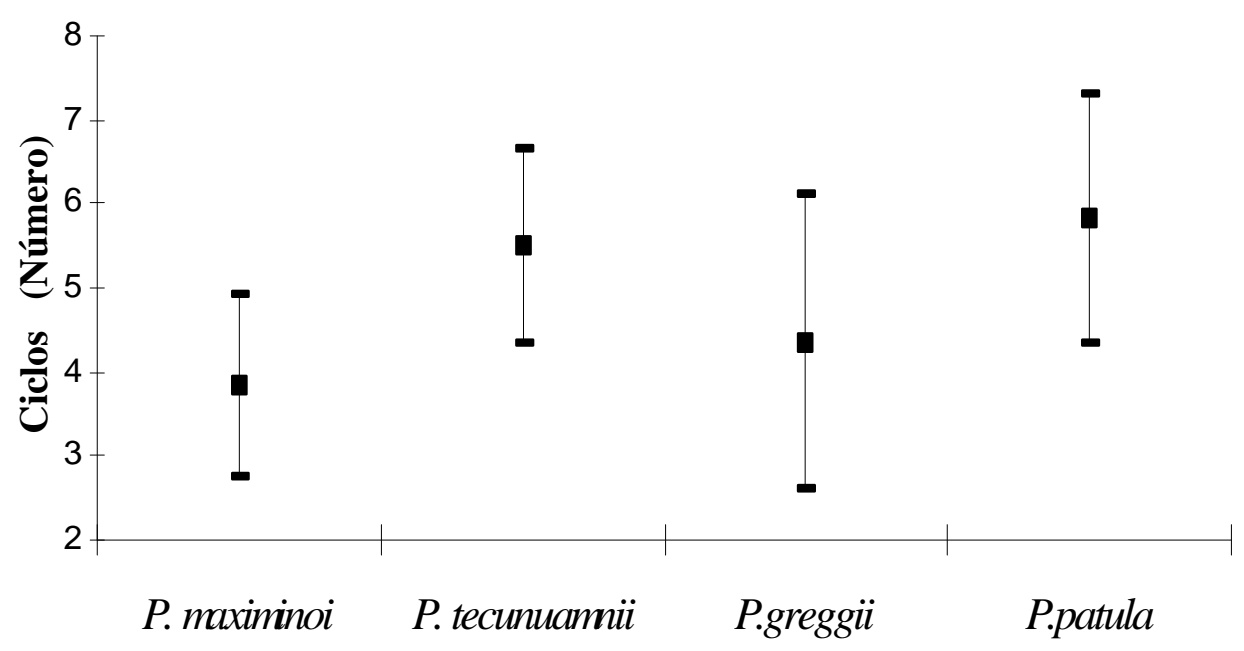

Figura 2. Valores promedios y desviaciones estándar por especie de las variables de crecimiento en altura y número de ciclos producidos. 
El número promedio anual de ciclos de crecimiento por especie varió desde 3.8 en Pinus maximinoi hasta 5.8 en $P$. patula, con diferencias entre ellas de más de $50 \%$ a favor de la segunda. Pinus greggii y $P$. tecunumanii presentaron 4.3 y 5.5 ciclos de crecimiento, respectivamente. Este comportamiento originó dos grupos distintivos de las especies; por un lado $P$. greggii y $P$. maximinoi, con una tendencia a desarrollar menor número de ciclos de crecimiento del brote terminal que $P$. patula y $P$. tecunumanii. Aunque no se encontraron datos para $P$. maximinoi y $P$. tecunumanii, López (1998) menciona un promedio de 3.3 ciclos de crecimiento al año (con una variación de 3.0 a 3.8 ciclos) en las procedencias sureñas de $P$. greggii y Gómez et al. (1998) encontraron un promedio de $4 \pm 1$ ciclos (con 2 a 6 ciclos por año) en $P$. patula. Estos valores promedio son menores que los encontrados en el presente estudio, pero el intervalo de variación es similar. En Pinus taeda, otra especie subtropical de Pinus nativa del Sureste de Estados Unidos, también se ha encontrado un promedio de cuatro ciclos de crecimiento por año (Allen y Wentworth, 1993).

La combinación de los datos anteriores muestran que las cuatro especies de pino evaluadas presentan diferentes patrones estructurales de crecimiento en altura. Por un lado, tenemos a $P$. maximinoi que muestra las mayores tasas de crecimiento en altura, con el menor número de ciclos de crecimiento del brote; por lo tanto, es la especie que presentó la mayor longitud promedio por ciclo $(68 \mathrm{~cm})$, que resultó 2 veces mayor que el promedio observado en $P$. patula, que se encuentra en el otro extremo $(33 \mathrm{~cm})$. Esto implica que $P$. maximinoi tiene fustes con un menor número de verticilos $\mathrm{y}$, por lo tanto, de ramas (mayor proporción de madera limpia), lo cual repercute favorablemente sobre la calidad de la madera, al reducir el número de zonas con presencia de nudos.
En el caso de Pinus tecunumanii, aunque tiene un crecimiento en altura muy cercano al de $P$. maximinoi, desarrolla un mayor número de ciclos de crecimiento del brote, reduciendo la longitud promedio de éstos $(46 \mathrm{~cm})$ hasta en un $32 \%$ con respecto a la longitud promedio observada en la especie anterior. Esto implica que aunque $P$. tecunumanii tiene un potencial similar al de $P$. maximinoi en el crecimiento inicial en altura, desarrolla un mayor número de verticilos, lo cual puede afectar la calidad de la madera.

Pinus greggii se ubicó en tercer lugar con respecto a la tasa de crecimiento en altura, pero con la formación de un número relativamente bajo de ciclos de crecimiento. Debido a lo anterior, la longitud promedio de los interverticilos fue mayor que en el caso de $P$. tecunumanii, con $39 \mathrm{~cm}$, lo cual significa que se puede obtener madera con un reducido número de nudos. En cambio, $P$. patula tuvo la menor tasa de crecimiento en altura con el mayor número de ciclos de crecimiento formados, lo que originó la menor longitud promedio de los interverticilos con $26 \mathrm{~cm}$. De mantenerse estas características, ésta especie tendría la menor producción de madera de las cuatro especies evaluadas, con una mayor cantidad de zonas con nudos.

En resumen, $P$. maximinoi y $P$. greggii alargaron más los ciclos de crecimiento como estrategia para lograr más crecimiento en altura, mientras que $P$. patula formó más ciclos de crecimiento para aumentar la altura de los árboles; en cambio, $P$. tecunumanii presentó una combinación de las dos estrategias anteriores porque formó más ciclos de crecimiento y alargó los ciclos de crecimiento lo más posible para lograr el crecimiento en altura.

\section{Variación intraespecífica.}

En Pinus greggii se encontró una amplia variación entre las procedencias en la tasa promedio de crecimiento en altura (Tabla 4). La procedencia de Valle Verde 
tuvo un crecimiento anual de $2.45 \mathrm{~m}, 32 \%$ más que el crecimiento promedio observado en el material procedente de Patoltecoya, que tuvo la menor tasa de crecimiento en esta especie. El número de ciclos formados durante el año de evaluación también varió ampliamente entre las poblaciones de $P$. greggii; la procedencia de El Madroño presentó el menor número de ciclos de crecimiento, mientras que la procedencia de Zimapán presentó el mayor número de éstos, casi un $41 \%$ más que en la primera.

Es interesante notar que a pesar de que Patoltecoya es la procedencia local, presentó la menor tasa de crecimiento con respecto a las demás. Aunque no existe información al respecto, es posible que esta menor tasa de crecimiento esté asociada con un mayor nivel de endogamia en esta población debido al reducido tamaño de la misma (Ramírez et al., 1997). Se ha encontrado que el aumento de la endogamia ocasiona una reducción en la tasa de crecimiento en altura de los árboles y en general del vigor de los individuos como en Pseudotsuga menziesii (Mirb.) Franco, Pinus ponderosa Dougl. ex. Laws. y Abies procera Rehd. (Sorensen y Miles, 1982), y en Pinus elliottii Engelm. var. elliottii (Matheson, et al., 1995).

En el caso de Pinus maximinoi es posible distinguir dos grupos de procedencias que presentan tasas de crecimiento similares entre sí (Tabla 5); en el primer grupo se encuentran las procedencias de Altamirano, San Jerónimo y San Juan Sacatepequez con una tasa de crecimiento aproximadamente $18 \%$ mayor que las procedencias del segundo grupo, que incluyó a San José Bayancuch y Tatumbla.

A diferencia del crecimiento en altura, el número de ciclos de crecimiento tuvo una mayor variación entre las procedencias de esta especie, con diferencias de casi dos ciclos entre las procedencias extremas. Con base en esta característica también es posible distinguir los dos grupos de procedencias mencionadas anteriormente, el grupo con mayor tasa de crecimiento en altura tuvo en promedio $25 \%$ menos ciclos de crecimiento que el segundo.

Tabla 4. Valores promedio y desviaciones estándar por procedencia de las variables de crecimiento en altura y número de ciclos producidos en Pinus greggii Engelm.

\begin{tabular}{||l|c|c||}
\hline PROCEDENCIAS & $\begin{array}{c}\text { CRECIMIENTO ANUAL EN } \\
\text { ALTURA }(\mathrm{m})\end{array}$ & $\begin{array}{c}\text { CICLOS DE CRECIMIENTO } \\
(\text { No. })\end{array}$ \\
\hline El Madroño, Qro. & $2.24 \pm 0.47$ & $3.63 \pm 1.83$ \\
Valle Verde, Qro. & $2.45 \pm 0.57$ & $4.24 \pm 1.40$ \\
Laguna Seca, Hgo. & $2.37 \pm 0.65$ & $4.42 \pm 2.00$ \\
San Joaquín, Qro. & $2.22 \pm 0.47$ & $5.03 \pm 2.17$ \\
El Piñón, Hgo. & $2.28 \pm 0.61$ & $3.74 \pm 1.68$ \\
Molango, Hgo. & $2.16 \pm 0.39$ & $4.73 \pm 2.00$ \\
Xocicoatlán, Hgo. & $2.22 \pm 0.57$ & $4.54 \pm 1.57$ \\
Cieneguilla, Hgo. & $2.14 \pm 0.58$ & $4.24 \pm 1.38$ \\
Zimapán, Hgo. & $2.03 \pm 0.38$ & $5.11 \pm 1.56$ \\
Patoltecoya, Pue. & $1.85 \pm 0.49$ & $3.88 \pm 1.28$ \\
\hline
\end{tabular}


Tabla 5. Valores promedio y desviaciones estándar por procedencia de las variables de crecimiento en altura y número de ciclos producidos en Pinus maximinoi $\mathrm{H}$. E. Moore

\begin{tabular}{||l|c|c||}
\hline \multicolumn{1}{|c|}{ PROCEDENCIAS } & $\begin{array}{c}\text { CRECIMIENTO } \\
\text { ANUAL EN ALTURA } \\
(\mathrm{m})\end{array}$ & $\begin{array}{c}\text { CICLOS DE CRECIMIENTO } \\
\text { (No.) }\end{array}$ \\
\hline Altamirano, Chis. & $2.64 \pm 0.58$ & $3.90 \pm 0.94$ \\
San Jerónimo, Oax. & $2.68 \pm 0.60$ & $3.24 \pm 0.95$ \\
San Juan Sacatepéquez, Guat. & $2.65 \pm 0.50$ & $3.09 \pm 0.71$ \\
Tatumbla, Fco. Morazán, Hond. & $2.23 \pm 0.50$ & $4.12 \pm 0.77$ \\
San José Bayancuch, Nicaragua & $2.28 \pm 0.64$ & $4.89 \pm 1.02$ \\
\hline
\end{tabular}

Pinus patula presentó una amplia variación entre procedencias en la tasa promedio de crecimiento en altura (Tabla 6), con valores en las procedencias extremas que van de 1.65 hasta $2.15 \mathrm{~m}$ en la procedencia de Tlahuelompan, lo que representa una diferencia entre ellas de 30 $\%$ a favor de la segunda. En el caso del número de ciclos de crecimiento desarrollados, se observó una variación similar, con diferencias de alrededor del 20 $\%$ entre las procedencias con valores promedio extremos, Tlaxco y Huayacocotla, (Tabla 6).
En el caso de Pinus tecunumanii se observó una mayor variación absoluta en el crecimiento en altura que en el caso de $P$. maximinoi, a pesar del número reducido de procedencias evaluadas, con diferencias cercanas a $20 \%$ entre los valores extremos; las dos procedencias de Centroamérica tuvieron mayores tasas de crecimiento en altura y menor número de ciclos de crecimiento que las del Sur de México (Tabla 7).

Tabla 6. Valores promedio y desviaciones estándar por procedencia de las variables de crecimiento en altura y número de ciclos producidos en Pinus patula Schl. et Cham.

\begin{tabular}{||l|c|c||}
\hline \hline PROCEDENCIAS & $\begin{array}{c}\text { CRECIMIENTO ANUAL EN } \\
\text { ALTURA }(\mathrm{m})\end{array}$ & $\begin{array}{c}\text { CICLOS DE CRECIMIENTO } \\
(\text { No. })\end{array}$ \\
\hline Pinal de Amoles, Qro. & $2.03 \pm 0.41$ & $5.64 \pm 1.39$ \\
Encarnación, Hgo. & $1.81 \pm 0.44$ & $5.54 \pm 1.65$ \\
Zacualtipan, Hgo. & $1.98 \pm 0.48$ & $6.16 \pm 1.30$ \\
Tlahuelompan, Hgo. & $2.15 \pm 0.52$ & $6.22 \pm 1.41$ \\
Huayacocotla, Ver. & $1.97 \pm 0.38$ & $6.38 \pm 1.13$ \\
Estación Apulco, Hgo. & $2.02 \pm 0.59$ & $6.15 \pm 1.52$ \\
Acaxochitlán, Hgo. & $1.87 \pm 0.51$ & $5.76 \pm 1.51$ \\
Ahuazotepec, Pue. & $1.83 \pm 0.48$ & $5.95 \pm 1.45$ \\
Zacatlán (Norte), Pue. & $2.03 \pm 0.41$ & $5.77 \pm 1.32$ \\
Zacatlán, Pue. & $1.74 \pm 0.43$ & $5.51 \pm 1.50$ \\
Tlaxco, Tlax. & $1.65 \pm 0.54$ & $5.08 \pm 1.61$ \\
\hline
\end{tabular}


Tabla 7. Valores promedio y desviaciones estándar por procedencia de las variables de crecimiento en altura y número de ciclos producidos en Pinus tecunumanii (Schw.)

Eguiluz et Perry.

\begin{tabular}{||l|c|c||}
\hline PROCEDENCIAS & $\begin{array}{c}\text { CRECIMIENTO ANUAL EN } \\
\text { ALTURA }(\mathrm{m})\end{array}$ & $\begin{array}{c}\text { CICLOS DE CRECIMIENTO } \\
(\text { No. })\end{array}$ \\
\hline Chanal, Chis. & $2.26 \pm 0.33$ & $6.03 \pm 0.94$ \\
Rancho Nuevo, Chis. & $2.26 \pm 0.43$ & $5.85 \pm 1.22$ \\
San Esteban, Honduras & $2.61 \pm 0.55$ & $4.97 \pm 0.78$ \\
Yucul, Matagalpa, Nicaragua & $2.73 \pm 0.62$ & $4.92 \pm 1.19$ \\
\hline
\end{tabular}

\section{Relación entre el número de ciclos y el crecimiento en altura.}

En el caso de Pinus greggii y $P$. maximinoiel crecimiento promedio en altura de las procedencias fue independiente del número de ciclos de crecimiento formados ( $r \leq 0.81$ n.s.). Por lo tanto, se pueden seleccionar las procedencias con mayor velocidad de crecimiento sin afectar en forma negativa la calidad de madera. En $P$. greggii estos resultados difieren de los encontrados por López (1998), quien menciona una correlación positiva entre la tasa de crecimiento en altura y el número de ciclos desarrollados, pero en ese caso la evaluación se hizo a nivel de familias en procedencias de diferentes regiones geográficas.

A diferencia de las especies anteriores, en $P$. patula se encontró una correlación positiva altamente significativa ( $p £ 0.01$ ) moderadamente elevada $(r=0.75)$ entre el crecimiento en altura y el número de los ciclos de crecimiento formados, lo cual implica que las procedencias de $P$. patula con mayor crecimiento en altura también forman más ciclos de crecimiento. Estos resultados contrastan con los de Vargas y Jacob (1993) quienes no encontraron una correlación significativa entre estas características, pero a nivel de familias de una misma población. Por otro lado, en especies como $P$. ponderosa Dougl. ex
Laws. y $P$. taeda L. se han encontrado correlaciones positivas entre estas dos características en edades tempranas (Read, 1980; Bridgewater et al., 1985).

En Pinus tecunumanii la correlación entre el crecimiento en altura y el número de ciclos formados también fue significativa $(p \leq 0.05)$, pero con signo negativo ( $r=-$ 0.98 ); esto indica que las procedencias que desarrollaron un mayor número de ciclos de crecimiento fueron las que tuvieron menor crecimiento promedio en altura, por lo que existen diferencias notorias entre ellas en el patrón de crecimiento en altura. Estos resultados son importantes puesto que ayudan a seleccionar las procedencias con mayor crecimiento en altura sin que se afecte en forma negativa la calidad de la madera, ya que también aumentará la proporción de madera sin nudos.

\section{Relación del crecimiento con el origen geográfico del germoplasma.}

Con excepción de Pinus maximinoi, en todas las especies evaluadas fue posible detectar un gradiente geográfico de variación asociado a las características de crecimiento en altura evaluadas. Sin embargo, en cada especie el patrón de variación se asoció en forma distintiva a coordenadas geográficas particulares (Tabla 8). 
Tabla 8. Correlaciones por especie entre las variables del crecimiento en altura, y las coordenadas de las procedencias

\begin{tabular}{|c|c|c|c|}
\hline & ESPECIE & CRECIMIENTO ANUAL & NUMERO DE CICLOS \\
\hline Latitud & $\begin{array}{l}P . \text { greggii } \\
(n=10) \\
P . \text { maximinoi }(n=5) \\
\text { P. patula } \\
(n=11) \\
P . \text { tecunumanii }(n=4)\end{array}$ & $\begin{array}{l}0.89 \text { ** } \\
0.76 \text { n.s. } \\
0.55 \text { n.s. } \\
-0.91 \text { n.s. }\end{array}$ & $\begin{array}{l}-0.10 \text { n.s. } \\
-0.54 \text { n.s. } \\
\\
0.43 \text { n.s. } \\
0.82 \text { n.s. }\end{array}$ \\
\hline Longitud & $\begin{array}{l}\text { P. greggii } \\
\text { P. maximinoi } \\
\text { P. patula } \\
\text { P. tecunumanii }\end{array}$ & $\begin{array}{l}0.58 \text { n.s. } \\
0.84 \text { n.s. } \\
0.19 \text { n.s. } \\
-0.98 \text { * }\end{array}$ & $\begin{array}{l}0.36 \text { n.s. } \\
-0.73 \text { n.s. } \\
0.02 \text { n.s. } \\
0.99 \text { ** }\end{array}$ \\
\hline Altitud & $\begin{array}{l}\text { P. Greggii } \\
\text { P. Maximinoi } \\
\text { P. Patula } \\
\text { P. Tecunumanii }\end{array}$ & $\begin{array}{l}0.16 \text { n.s. } \\
0.12 \text { n.s. } \\
-0.82 \text { ** } \\
-0.98 \text { * }\end{array}$ & $\begin{array}{l}0.27 \text { n.s. } \\
-0.48 \text { n.s. } \\
-0.80 \text { ** } \\
0.99 * *\end{array}$ \\
\hline
\end{tabular}

En Pinus greggii la tasa de crecimiento promedio anual de las procedencias se correlacionó en forma positiva $(r=0.89)$ con la latitud del sitio de origen de las procedencias (Tabla 8). Estos resultados podrían parecer contradictorios, ya que es bien conocido que las poblaciones de la parte Norte del área de distribución natural tienen una tasa menor de crecimiento en altura que las del Sur (Donahue y López, 1996; Ramírez et al., 1997; López 1998); sin embargo, en este estudio sólo se incluyeron poblaciones de la región Sur.

En cambio, en Pinus patula la tasa de crecimiento en altura $(r=-0.82)$ y el número de ciclos $(r=-0.80)$ se asociaron en forma negativa con la altitud. Esto implica que las procedencias originarias de sitios más bajos presentaron mayor crecimiento en altura y desarrollaron mayor número de ciclos de crecimiento. Este tipo de relación entre el crecimiento en altura y la altitud del sitio de origen también se ha encontrado en otras especies como $P$. ponderosa Laws. y $P$. jeffreyi Grev. \& Balf. en evaluaciones hechas a diferentes edades (Callaham y Metcalf, 1959).
Por otro lado, en $P$. tecunumanii la tasa de crecimiento en altura $(r=-0.98)$ y el número de ciclos de crecimiento $(r=0.99)$ se asociaron en forma significativa con la longitud y la altitud del sitio de origen de las poblaciones pero con signo contrario (Tabla 8). Sin embargo, debido al reducido número de poblaciones de esta especie incluidas en el estudio, es necesario tomar estos valores con reservas, ya que la correlación podría modificarse drásticamente al incluir una muestra de mayor tamaño.

\section{CONCLUSIONES}

Los resultados del presente trabajo muestran que existe una variación significativa en el crecimiento en altura y en el número de ciclos de crecimiento formados tanto entre especies como dentro de ellas. La variación interespecífica fue de 2 a 6 veces mayor que la variación intraespecífica, por lo que existen diferencias notorias en el potencial productivo y en el patrón estructural de crecimiento del brote entre las especies evaluadas. La interacción genotipo*ambiente no fue significativa, lo 
cual implica que tanto a nivel de especies como de las procedencias el comportamiento fue estable en los dos sitios de plantación.

A nivel de especies, en Pinus maximinoi se observó la mayor tasa de crecimiento en altura ( $2.50 \mathrm{~m}$ por año), con el menor numero de ciclos de crecimiento (3.8 por año); en $P$. tecunumanii se observó una tasa de crecimiento en altura similar a la de la especie anterior (2.44 m por año), pero desarrolló un mayor número de ciclos de crecimiento (5.5 por año); en $P$. patula se observó la menor tasa de crecimiento en altura de todas las especies evaluadas (1.92 m por año), y el mayor número de verticilos (5.8 por año) por lo que presentó la menor longitud promedio de éstos, remarcando las diferencias entre especies en el patrón estructural de desarrollo del brote terminal.

En todas las especies se observaron diferencias en la variación entre las procedencias en la tasa de crecimiento en altura de entre 20 y $50 \mathrm{~cm}$ por año. Sin embargo, en cada especie se encontraron patrones diferentes de asociación entre esta característica y el número de ciclos de crecimiento. En $P$. patula, el crecimiento promedio de las procedencias se correlacionó en forma positiva con el número de ciclos de crecimiento, mientras que en $P$. tecunumanii la correlación fue negativa y en $P$. maximinoi y $P$. greggii la correlación no fue significativa.

Las especies evaluadas también presentaron diferentes patrones de variación geográfica en las características de crecimiento del brote terminal. En $P$. greggii la tasa de crecimiento en altura se asoció en forma positiva con la latitud del sitio de origen del germoplasma, mientras que en $P$. patula la tasa de crecimiento en altura y el número de ciclos de crecimiento se asoció en forma negativa con la altitud del sitio de origen, y en $P$. maximinoi no se encontró ningún patrón geográfico significativo.

\section{AGRADECIMIENTOS}

Los autores agradecen profundamente al Sr. Juan Ramón Fernández Alonso, propietario de la finca "Los Ayacahuites", el apoyo proporcionado para el establecimiento del ensayo y la toma de datos. El germoplasma de Pinus maximinoi y $P$. tecunumanii fue proporcionado por CAMCORE. Se agradece el financiamiento parcial del CONACYT como parte del proyecto P220-CCOR-904153.

\section{REFERENCIAS}

Allen, H.L. y T.R. Wentworth. 1993. Vegetation control and site preparation affect patterns of shoot elongation for 3 -year-old loblolly pine. Can. J. For. Res. 23(10): 2110-2115.

Bridgewater, F. E., C.G. Williams y R.G. Campbell. 1985. Patterns of leader elongation in loblolly pine families. For. Sci. 31(4): 933-944.

Callaham, R. Z. y W. Metcalf. 1959. Altitudinal races of Pinus ponderosa confirmed. J. of For. 57: 500-502.

Cannell, M. G. R., S. Thompson y R. Lines. 1976. An analysis of inherent differences in shoot growth within some north temperate conifers. In: Cannell, M.G.R. y F.T. Last. (Eds.) 1976. Tree physiology and yield improvement. Academic Press. London. p: 173-206.

Daniels, P. W., U. E. Helms y F. S. Baker. 1982. Principios de silvicultura. Editorial McGraw-Hill. México. 492 p.

Donahue, J.K. y J. López U. 1996. Geographic variation in leaf, cone and seed morpholgy of Pinus greggii native forests. Forest Ecol. Manag. 82: 1-3.

Dvorak, W. S. y J. K. Donahue 1988. Pinus maximinoi seed collections in Mexico 
and Central America. CAMCORE. Bull. on Tropical Forestry No. 4. North Carolina State University. $47 \mathrm{p}$.

Dvorak, W. S. y J. K. Donahue. 1992. Research review 1980-1992. Central American and Mexico Coniferous Resources Cooperative. Raleigh, N.C. U.S.A. pp: 37-52.

Dvorak,W. S., J. K. Donahue y J. A. Vasquez. 1995. Early performance of CAMCORE introductions of Pinus patula in Brazil, Colombia and South Africa. South African For. Journal. 174: 23-33.

Dvorak, W. S., J. E. Kietzka y J. K. Donahue. 1996. Three-year survival and growth of provenances of Pinus greggii in the tropics and subtropics. For. Ecol. Manag. 83(1-2): 123-131.

García, E. 1981. Modificaciones al sistema de clasificación climática de Köppen. Ed. Enriqueta García de Miranda. México. $241 \mathrm{p}$.

Gómez C.,M., J.J. Vargas H., J. Jasso M., A. Velázquez M. y C. Rodríguez F. 1998. Patrón de crecimiento anual del brote terminal en árboles jovenes de Pinus patula. Agrociencia 32(4): 357363.

Loock, E. E. M. 1950. The Pines of Mexico and British Honduras. South Africa Department of Forestry Bulletin 35.

López A., J.L. 1998. Variación intraespecífica en el patrón de crecimiento en altura del brote terminal en Pinus greggii Engelm. Tesis Profesional, Universidad Nacional Autónoma de México. 62 p.

Matheson, A. C., T. L. White y G. R. Powell. 1995. Effects of inbreeding on growth, stem form and rust resistance in Pinus elliottii. Silvae Genetica. 44(1): 37-46.

Perry, J. P. Jr. 1991. The pines of Mexico and central America. Timber Press. Portland, Oregon E.U.A. 231 p.

Ramírez H., C., J. J. Vargas H., J. Jasso M., G. Carrillo C. y H. Guillén A. 1997. Variación isoenzimática en diez poblaciones naturales de Pinus greggii Engelm. Agrociencia 31(2): 223-229.

Read, R. A. 1980. Genetic variation in seedling progeny of Ponderosa pine provenances. For. Sci. Monograph No. 23. $59 \mathrm{p}$.

Sorensen, F. C. y R. S. Miles. 1982. Inbreeding depression in height, height growth, and survival of Douglas-fir, ponderosa pine, and noble fir to 10 years of age. For. Sci. 28: 283-293.

Vargas H., J. J. y V. Jacob C. 1993. Variación genética en la elongación del brote terminal de Pinus patula. Resúmenes de Ponencias del Primer Congreso Mexicano Sobre Recursos Forestales. Saltillo, Coah., Méx. p. 94.

Wormald, T. J. 1975. Pinus patula. Unit of Tropical Silviculture Dept. of Forestry. England. $217 \mathrm{p}$.

Wright, J. A. y L. F. Osorio. 1992. Results of provenance and family within provenance trials of Pinus tecunumanii in Colombia, South America. For. Ecol. and Manag. 55: 107-116.

Zobel, B. J. y J. P. Van Buijtenen. 1989. Wood variation Its causes and control. Springer-Verlag, Berlin Heidelberg New York, Germany. 363 p.

Zobel, B.J. y J. Talbert. 1988. Técnicas de mejoramiento genético de árboles forestales. Editorial Limusa, México. $545 \mathrm{p}$. 